\title{
Energy and multipartite entanglement in multidimensional and frustrated spin models
}

\author{
Otfried Gühne ${ }^{1}$ and Géza Tóth ${ }^{2,3}$ \\ ${ }^{1}$ Institut für Quantenoptik und Quanteninformation, \\ Österreichische Akademie der Wissenschaften, A-6020 Innsbruck, Austria \\ ${ }^{2}$ Max-Planck-Institut für Quantenoptik, Hans-Kopfermann-Straße 1, D-85748 Garching, Germany \\ ${ }^{3}$ Research Institute of Solid State Physics and Optics, \\ Hungarian Academy of Sciences, H-1525 Budapest P.O. Box 49, Hungary
}

(Dated: August 20, 2018)

\begin{abstract}
We investigate the relation between the entanglement properties of a quantum state and its energy for macroscopic spin models. To this aim, we develop a general method to compute energy bounds for states without certain forms of multipartite entanglement. Violation of these bounds implies the presence of these types of multipartite entanglement. As examples, we investigate the Heisenberg model in different dimensions, the Ising model and the XX model in the presence of a magnetic field. Finally, by studying the Heisenberg model on a triangular lattice, we demonstrate that our techniques can be applied also to frustrated systems.
\end{abstract}

PACS numbers: 03.65.-w, 03.67.-a, 05.30.-d

\section{INTRODUCTION}

In recent years, the investigation of entanglement in condensed matter systems has become one of the main lines of research in quantum information science [1, 2, 3]. The increased interest in this topic is fed by several motivations. On the one hand, the studies helped to understand fundamental properties of condensed matter systems like quantum phase transitions. On the other hand, they lead to results of practical importance since they allowed to design new simulation techniques for the calculation of ground state energies of spin models [4].

What kinds of entanglement occur in natural situations? This question provides another motivation for studying entanglement properties of condensed matter systems. Indeed, condensed matter systems and especially spin models are natural candidates for our studies where various forms of entanglement might occur, mainly at low temperatures. One possibility to study the presence of entanglement in spin systems is to relate the energy or other macroscopic observables of the system to certain entanglement properties of the state [5, 6, 7, 8, 9, 10].

In this paper we attempt to proceed in this direction by investigating the relation between the energy of a state and its multipartite entanglement properties. We will derive a general method for calculating energy thresholds for states without certain types of multipartite entanglement. Below these energies, and consequently below a certain temperature, the state must therefore contain multipartite entanglement. Our approach is, however, not restricted to states in thermal equilibrium. We demonstrate that our method can successfully be applied to various models and also to frustrated systems. In this way, we extend the results of Ref. [10] where such energy thresholds have been computed for two special spin models in one dimension.

Our paper is divided into four sections. These are organized as follows. In Section II we introduce the notion of multipartite entanglement that we use in this paper. That is, we explain the definition of $k$-producibility. We also pose the problem that we want to solve. In Section III we present our method for computing the desired energy bounds. We present in detail the calculation for a two-dimensional Heisenberg model on a square lattice, the generalization to other models is then straightforward. In Section IV we discuss three simple applications: the Heisenberg model in various dimensions, and the Ising model and the XX model with a magnetic field in one dimension. For the Ising model, we also discuss the impact of phase transitions on our energy thresholds. In Section $\mathrm{V}$ we consider the Heisenberg model a twodimensional triangular lattice. We show that with some modifications our methods can also be used to investigate multipartite entanglement in such a frustrated system.

\section{DEFINITIONS AND STATEMENT OF THE PROBLEM}

Let us first explain the notion of multipartite entanglement that we use for our study. This is the so-called $k$-producibility, introduced in Ref. [10]. It is defined as follows: For a pure state $|\psi\rangle$ on $N$ qubits we ask whether it is possible to write

$$
|\psi\rangle=\left|\phi_{1}\right\rangle \otimes\left|\phi_{2}\right\rangle \otimes \ldots \otimes\left|\phi_{K}\right\rangle
$$

where the $\left|\phi_{i}\right\rangle$ are states of maximally $k$ qubits. If this is the case, then only $k$-qubit entanglement is necessary to generate $|\psi\rangle$ and the state $|\psi\rangle$ does not contain any $(k+1)$-qubit entanglement. If Eq. (11) holds, we call the state $k$-producible, if not, we say that $|\psi\rangle$ contains $(k+1)$-partite entanglement. Examples of four- and twoproducible states are shown in Figs. 1 and 2.

For mixed states, we can extend this definition via considering convex combinations. I.e., we call a mixed state 


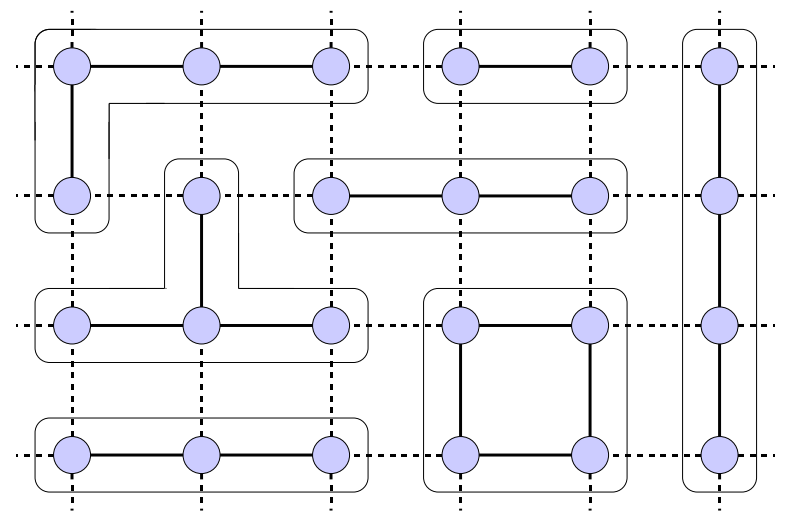

FIG. 1: Schematic view of a four-producible state in a spin model of 24 qubits. Dashed lines correspond to interactions between disentangled qubits and solid lines represent the interactions between qubits which are allowed to be entangled.

$\varrho k$-producible if we can write

$$
\varrho=\sum_{i} p_{i}\left|\psi_{i}\right\rangle\left\langle\psi_{i}\right|
$$

with $p_{i} \geq 0, \sum_{i} p_{i}=1$ and $k$-producible $\left|\psi_{i}\right\rangle$. If this is not the case, $\varrho$ contains $(k+1)$-partite entanglement. Physically, a $k$-producible mixed state requires $k$-qubit entanglement and mixing for its creation only. Conversely, a state contains $k+1$-party entanglement if and only if the quantum correlations of this state cannot be explained by assuming $k$-qubit entanglement only.

This classification of multipartite entanglement has some connections to the usual notion of $k$-separability, which is often used for small numbers of qubits. This issue has been discussed in Ref. [10]. Here, we only want to point out that the $N$-separable (fully separable) states are by definition the states which can be written as $\varrho=\sum_{i} p_{i} \varrho_{1} \otimes \ldots \otimes \varrho_{N}$. These states are just the oneproducible states.

The notion of $k$-producibility leads to a discrete classification of multipartite states. For pure states, it is easy to see that the $k$-producible states form a set of measure zero in the set of $2 k$-producible states and that in the vicinity of any $k$-producible state one can find states with arbitrary high producibility [11]. For mixed states, however, this is not true anymore, and one can show (as for the notion of $k$-separability [12]) that the set of mixed $k$-producible states is not of measure zero in the space of all mixed states.

Finally, it is worth noting that the notion of $k$ producibility for pure states has a close relation to the Schmidt measure, which is an entanglement monotone for multi-qubit states [13]. The Schmidt number of a pure multi-qubit state is defined as follows. One expands $|\psi\rangle$ as the sum of tensor products of single qubit states $|\psi\rangle=\sum_{k=1}^{R}\left|\phi_{k}^{(1)}\right\rangle \otimes\left|\phi_{k}^{(2)}\right\rangle \otimes\left|\phi_{k}^{(3)}\right\rangle \otimes \ldots$ For every quantum state we take the expansion with the minimal $R$, which will be denoted by $r$. Then $\log _{2}(r)$ is the Schmidt measure of $|\psi\rangle$ and for N-qubit quantum states we have always $\log _{2}\left(r_{q}\right) \leq N$.

Since the Schmidt number for two-qubit states in maximally two and for three-qubit states is maximally three [13], we can conclude that for one-, two- and threeproducible states

$$
\begin{aligned}
& \log _{2} r_{1 p}=0 \\
& \log _{2} r_{2 p} \leq \frac{N \log _{2}(2)}{2}=\frac{N}{2} \\
& \log _{2} r_{3 p} \leq \frac{N \log _{2}(3)}{3} \approx 0.53 N
\end{aligned}
$$

holds.

Now we can state the main problem we want to study in this paper. Let us assume that we have a macroscopic spin system of qubits on some lattice, which interact via some Hamiltonian

$$
H=\sum_{<i, j>} h_{i j}
$$

which is a sum of two-qubit interactions. We always assume periodic boundary conditions. For this situation, we want to derive lower bounds for $\langle H\rangle$ for $k$-producible states. That is, we want to compute a constant $E_{k p}$ such that

$$
\langle H\rangle \geq E_{k p}
$$

holds for all $k$-producible states. If this bound is then violated at low temperatures, the state under consideration contains $(k+1)$-party entanglement. Note, however, that we do not restrict our attention to states in thermal equilibrium. Since we assume that the number of qubits $N$ is large, it will be convenient to express $E_{k p}$ as a rescaled energy per interaction bond.

In the next Section, we will present the main idea of our method to compute $E_{k p}$.

\section{ESTIMATING THE ENERGY}

In this Section, we present as the main result of the paper a general method to estimate the energy for $k$ producible states. While the main result is quite simple, its proof requires some technical effort. The logical structure is as follows: For a pure $k$-producible state some expectation values in the Hamiltonian factorize and some not. We collect all the factorizing terms in the Hamiltonian, and estimate them via the Cauchy-Schwarz inequality. Then, we arrive at Eqs. (13] 14). If we can perform the maximization in Eq. (13) then the Eq. (14) delivers the desired energy bound. The point is that even for macroscopic $k$-producible states the maximization requires only a maximization over $k$-qubit states. Sometimes, this can be done analytically, otherwise it can be solved numerically in an efficient manner. Finally we discuss whether the derived bounds are sharp. 


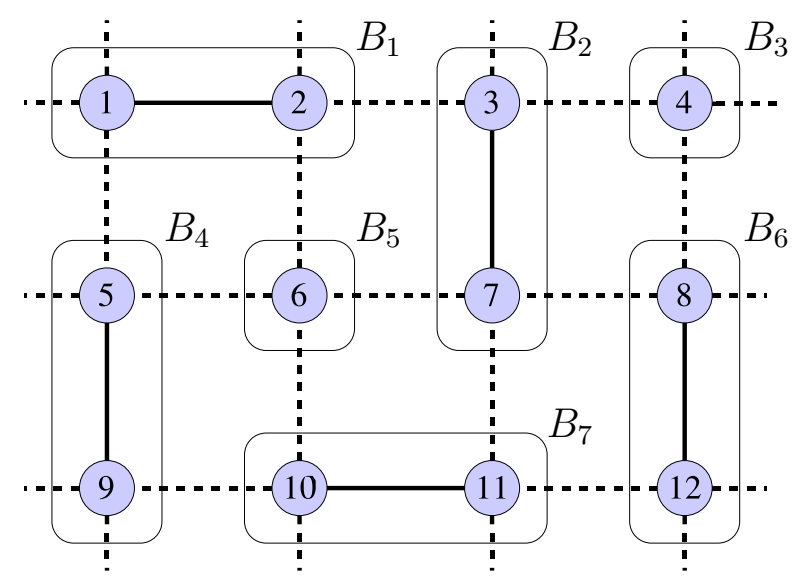

FIG. 2: A possible grouping for a pure two-producible state in twelve-qubit spin system. See text for details.

So let us explain our method in the following example. We want to derive a bound for two-producible states for a two-dimensional Heisenberg lattice. That is, we consider the Hamiltonian

$$
\begin{aligned}
H_{H} & =\sum_{<i, j>} h_{i j}, \\
h_{i j} & =X_{i} X_{j}+Y_{i} Y_{j}+Z_{i} Z_{j} .
\end{aligned}
$$

for a two-dimensional system with periodic boundary conditions. Here and in the following, $X_{i}, Y_{i}, Z_{i}$ denote the Pauli matrices $\sigma_{x}^{(i)}, \sigma_{y}^{(i)}, \sigma_{z}^{(i)}$, acting on the $i$-th qubit. We will exemplify the definitions required for our method using the example of a special two-producible state of twelve qubits given in Fig. 2.

In order to bound the energy for two-producible states, it suffices to consider a generic pure two-producible state $|\psi\rangle$. This comes from the fact that the mixed $k$-producible states form a convex set in the state space and the pure $k$-producible states are its extremal points. Thus, any linear function takes its maximum in a pure state as an extremal point.

A fixed two-producible $|\psi\rangle$ results in a partition of the whole spin system into several one- and two-qubit blocks. Indeed, one can identify some pairs $i, j$ of qubits where the reduced state is allowed to be entangled, and some single qubits $k$, which are not entangled with any other qubit. Let us denote the total number of blocks by $K$, the number of one-qubit blocks by $L_{1}$ and the number of two-qubit blocks by $L_{2}$. A possible blocking is shown in Fig. 2: We have seven blocks $B_{1}, \ldots, B_{7}$, where $B_{3}$ and $B_{5}$ are single-qubit blocks and the rest are two-qubit blocks. Thus we have $K=7, L_{1}=2$, and $L_{2}=5$. It is important to note that we can restrict our attention to the case where the two-qubit blocks are between interacting qubits. This is true for the following reason: If a two-qubit block consists of two noninteracting qubits $i, j$, then the Hamiltonian is only sensitive to the reduced density matrices at each qubit, i.e., it does only take then local properties into account. Thus, we can replace this two-qubit block by two one-qubit blocks.
In general, the mean value of the Hamiltonian consists of two-qubit expectation values of the type $W_{i j}=$ $\left\langle A_{i} A_{j}\right\rangle, A=X, Y, Z$. For simplicity, we denote in the following $\left\langle A_{i} B_{j}\right\rangle=a_{i} b_{j}$ and $\left\langle A_{i}\right\rangle=a_{i}$ for $A, B=X, Y, Z$. Note that this definition implies that in general $a_{i} b_{j} \neq$ $a_{i} \cdot b_{j}$ and these notations have to be distinguished. Due to the special partition, however, some of the mean values factorize. For instance, in the situation of Fig. 2 we have $x_{2} x_{3}=x_{2} \cdot x_{3}$. Now we have to find an efficient way for taking all these contributions into account.

For this purpose, we define for each block $B_{i}$ three sets of indices: $I(i)$ are the qubits which lie inside $B_{i}, R(i)$ are the qubits inside $B_{i}$ which interact via the Hamiltonian with qubits outside of $B_{i}$ and $N(i)$ are the qubits outside $B_{i}$, which nevertheless interact with some qubit of $I(i)$. For instance, in the example of Fig. 2 we have $I(1)=$ $\{1,2\}, R(1)=\{1,2\}$ and $N(1)=\{3,4,5,6,9,10\}$. For our special case of a two-producible state we have $I(i)=$ $R(i)$, but in general this does not have to be the case.

Now we can define for each block a set of expectation values from the Hamiltonian in the following way. We define

$$
\mathcal{I}(i):=\left\{W_{k l} \mid k, l \in I(i)\right\}
$$

as the contributions of the Hamiltonian inside the block $B_{i}$. In our example, this would be $\mathcal{I}(1)=$ $\left\{x_{1} x_{2}, y_{1} y_{2}, z_{1} z_{2}\right\}$. Note that the set $\mathcal{I}(i)$ is empty for the one-qubit blocks. In the following, we will denote the single elements of sets like $\mathcal{I}(i)$ by $\mathcal{I}(i)[j]$.

Then, we collect the "outgoing" contributions from a block via all the two-qubit Hamiltonians. That is, we define:

$$
\mathcal{R}(i):=\left\{\left.\left(W_{k l}\right)\right|_{k} \mid k \in R(i), l \in N(i)\right\} .
$$

Here, using $\left.\right|_{k}$ with $k \in R(i)$ we express that the twoqubit contributions are restricted to the operator acting only on the qubit belonging to $R(i)$. For example, from a term of the form $W_{23}=\left\langle X_{2} X_{3}\right\rangle$ we take only the term $\left\langle X_{2}\right\rangle=x_{2}$. Also, we take all terms with their respective multiplicity, i.e., if the qubit $k \in R(i)$ interacts with several qubits in $N(i)$, the same term appears several times in $\mathcal{R}(i)$. For our example in Fig. 2 we would have: $\mathcal{R}(1)=$ $\left\{x_{1}, y_{1}, z_{1}, x_{1}, y_{1}, z_{1}, x_{1}, y_{1}, z_{1}, x_{2}, y_{2}, z_{2}, x_{2}, y_{2}, z_{2}, x_{2}, y_{2}\right.$, $\left.z_{2}\right\}$.

Finally, we count for each block the contributions in the neighborhood via:

$$
\mathcal{N}(i):=\left\{\left.\left(W_{k l}\right)\right|_{l}, \quad \mid k \in R(i), l \in N(i)\right\} .
$$

These are, in a certain sense, the complementary contributions to the contributions in $\mathcal{R}(i)$. We always write them in the same order as the contributions in $\mathcal{R}(i)$, i.e. the first element of $\mathcal{R}(i)$ should correspond to the first element of $\mathcal{N}(i)$ in the Hamiltonian, etc. In our example, we would have: $\mathcal{N}(1)=$ $\left\{x_{9}, y_{9}, z_{9}, x_{4}, y_{4}, z_{4}, x_{5}, y_{5}, z_{5}, x_{10}, y_{10}, z_{10}, x_{3}, y_{3}, z_{3}, x_{6}\right.$, $\left.y_{6}, z_{6}\right\}$. The idea behind these definitions of $\mathcal{R}(i)$ and $\mathcal{N}(i)$ is the following: The terms in $\mathcal{R}(i)$ and $\mathcal{N}(i)$ 
are just the ones which factorize in the Hamiltonian. Thus, viewing $\mathcal{R}(i)$ and $\mathcal{N}(i)$ as vectors, the scalar product corresponds to the mean value of some terms in the Hamiltonian, $\mathcal{R}(i) \cdot \mathcal{N}(i)=\sum_{k} \mathcal{R}(i)[k] \quad \mathcal{N}(i)[k]=$ $\sum_{k \in B(i), l \notin B(i)}\left\langle W_{k l}\right\rangle$.

To estimate $\langle H\rangle$ for a given two-producible state $|\psi\rangle$ we interpret $\mathcal{R}(i)$ and $\mathcal{N}(i)$ as real vectors. We then define

$$
\begin{aligned}
& \vec{v}_{1}:=\frac{1}{\sqrt{2}} \cdot \mathcal{R}(1) \oplus \ldots \oplus \frac{1}{\sqrt{2}} \cdot \mathcal{R}(K), \\
& \vec{v}_{2}:=\frac{1}{\sqrt{2}} \cdot \mathcal{N}(1) \oplus \ldots \oplus \frac{1}{\sqrt{2}} \cdot \mathcal{N}(K)
\end{aligned}
$$

Please note that a term of the type $W_{k l}=x_{k} x_{l}=x_{k} \cdot x_{l}$ originating from an interaction between two blocks $B_{i}$ and $B_{j}$ appears twice in each of these vectors: one time with $x_{k} \in \mathcal{R}(i)$ and $x_{l} \in \mathcal{N}(j)$ and one time with $x_{k} \in$ $\mathcal{N}(i)$ and $x_{l} \in \mathcal{R}(j)$. Thus, $\vec{v}_{1}$ and $\vec{v}_{2}$ are built of the same terms, but in different order. This implies that $\left\|\vec{v}_{1}\right\|=\left\|\vec{v}_{2}\right\|$.

With this definition, it follows that

$$
\langle H\rangle=\sum_{i=1}^{K} \sum_{k} \mathcal{I}(i)[k]+\vec{v}_{1} \cdot \vec{v}_{2}
$$

holds. This implies due to the Cauchy-Schwarz inequality that

$$
\begin{aligned}
\langle H\rangle & \geq \sum_{i=1}^{K} \sum_{k} \mathcal{I}(i)[k]-\left\|\vec{v}_{1}\right\|\left\|\vec{v}_{2}\right\| \\
& =-\left(\sum_{i=1}^{K} \sum_{k}-\mathcal{I}(i)[k]+\left\|\vec{v}_{1}\right\|^{2}\right) .
\end{aligned}
$$

The key point is that the right hand side of this inequality can be estimated by minimization for each of the blocks $B_{i}$ separately. Indeed, if we define for the block $B_{i}$

$$
C_{i}:=\max _{|\psi\rangle}\left[\sum_{k}-\mathcal{I}(i)[k]+\frac{1}{2} \sum_{k}(\mathcal{R}(i)[k])^{2}\right],
$$

where $|\psi\rangle$ is a quantum state on the block $B_{i}$, we have

$$
\langle H\rangle \geq-\sum_{i} C_{i}
$$

The estimation of the $C_{i}$ does now only depend on the fact whether the block $B_{i}$ is a one- or a two-qubit block and not on the relations between these blocks. For the Heisenberg interaction, we have

$$
C_{i}=\max _{|\psi\rangle}\left[2\left(x_{k}^{2}+y_{k}^{2}+z_{k}^{2}\right)\right]=2
$$

for a one-qubit block $B_{i}$ on the qubit $k$ and

$$
\begin{aligned}
C_{i}= & \max _{|\psi\rangle}\left[-x_{k} x_{l}-y_{k} y_{l}-z_{k} z_{l}+\right. \\
& \left.+\frac{3}{2}\left(x_{k}^{2}+y_{k}^{2}+z_{k}^{2}+x_{l}^{2}+y_{l}^{2}+z_{l}^{2}\right)\right]=\frac{13}{3}
\end{aligned}
$$

for a two-qubit block on the qubits $k$ and $l$. This bound can be obtained from the representation of $|\psi\rangle\langle\psi|=$ $\sum_{k, l=1, x, y, z} \lambda_{k l} \sigma_{k} \otimes \sigma_{l}[10]$. It is also a special case of a general bound presented as Lemma 1 in the Appendix.

With these bounds, we immediately get for our example in Fig. 2 the bound $\left\langle H_{H}\right\rangle \geq-(5 \cdot 13 / 3+2 \cdot 2)=-77 / 3$. For the general case of $N$ qubits, we get

$$
\left\langle H_{H}\right\rangle \geq-\max _{L_{1}+2 L_{2}=N}\left[2 \cdot L_{1}+\frac{13}{3} \cdot L_{2}\right] \geq-\frac{13 N}{6} \approx-2.16 N .
$$

Since a two-dimensional lattice of $N$ qubits has $2 N$ bonds, the energy per bond for two-producible states is bounded from below by

$$
\frac{\left\langle H_{H}\right\rangle}{2 N} \geq E_{2 p}=-\frac{13}{12}
$$

Two questions arise at this point. First, we have to ask whether this bound is useful, in the sense that it is violated at low temperatures. This is the case since for the ground state the energy per bond is $E_{0}=-1.338$ 7, 14]. Thus, in a considerable temperature regime, the thermal states cannot be two-producible.

Second, the question arises whether the derived bound is sharp. This question deserves some discussion. The idea to show sharpness of an obtained bound is the following: Let us assume we have found states $\left|\phi_{i}\right\rangle$ for which the maxima $C_{i}$ in Eq. (13) are obtained. Then we have to build out of these states $\left|\phi_{i}\right\rangle$ the total state $|\psi\rangle$ such that $|\psi\rangle$ saturates the bound in Eq. (14). To do so, we have to assure, that for the state $|\psi\rangle$ the Cauchy-Schwarz inequality in Eq. (12) was sharp, i.e. $\vec{v}_{1} \cdot \vec{v}_{2}=-\left\|\vec{v}_{1}\right\|\left\|\vec{v}_{2}\right\|$. This can be done in two steps: First we guarantee that $\vec{v}_{1} \cdot \vec{v}_{2}=\left\|\vec{v}_{1}\right\|\left\|\vec{v}_{2}\right\|$. Then, by applying some unitary transformations on the $\left|\phi_{i}\right\rangle$ we make sure that $\vec{v}_{1} \cdot \vec{v}_{2}=-\left\|\vec{v}_{1}\right\|\left\|\vec{v}_{2}\right\|$.

Let us show how this works in our example of twoproducible states [15]. Let $|\phi\rangle_{k, l}$ be the state saturating Eq. (13) on the qubits $k, l$. Let us enumerate the qubits as in Fig. 2. and consider the total state $|\tilde{\psi}\rangle=|\phi\rangle_{1,2} \otimes\left|\phi^{\prime}\right\rangle_{3,4} \otimes|\phi\rangle_{5,6} \otimes\left|\phi^{\prime}\right\rangle_{7,8} \otimes|\phi\rangle_{9,10} \ldots$ Here, $\left|\phi^{\prime}\right\rangle=\mathcal{S}(|\phi\rangle)$ is the state $|\phi\rangle$ where the qubits are swapped. This construction implies that the reduced states of $|\tilde{\psi}\rangle$ on the qubits $1,4,5,8 \ldots$ are identical, as well as the reduced states on the qubits $2,3,6,7 \ldots$ Since the corresponding reduced states are identical, the factorizing terms between two qubits (say, 2 and 3 ) are just squares of some expectation values, hence $\vec{v}_{1}$ and $\vec{v}_{2}$ are parallel and $\vec{v}_{1} \cdot \vec{v}_{2}=\left\|\vec{v}_{1}\right\|\left\|\vec{v}_{2}\right\|$.

To perform the second step, note that the state $|\phi\rangle_{k, l}$ on the qubits $k, l$ gives rise to some sign distribution of the expectation values $x_{k}, y_{k}, z_{k}$ and $x_{l}, y_{l}, z_{l}$. Then we define $\left|\phi^{\prime \prime}\right\rangle$ as follows. We first swap, i.e. $\left|\phi^{\prime \prime}\right\rangle_{m, n}=$ $\left|\phi^{\prime}\right\rangle_{m, n}=\mathcal{S}\left(|\phi\rangle_{m, n}\right)$, then, by local unitary transformations, we flip the signs of $x_{m}, z_{m}$ and $x_{n}, z_{n}$ on the qubits $m, n$. Finally, we transpose the density matrix of the state, which flips also the signs of $y_{m}$ and $y_{n}$. Thus we have finally $a_{k}=-a_{n}$ and $a_{l}=-a_{m}$ for $a=x, y, z$ and 
$\left|\phi^{\prime \prime}\right\rangle$. Note that $\left|\phi^{\prime \prime}\right\rangle$ still saturates Eq. (16), since the expectation values $a_{m} a_{n}$ are not affected. Then, defining $|\psi\rangle=|\phi\rangle_{1,2} \otimes\left|\phi^{\prime \prime}\right\rangle_{3,4} \otimes|\phi\rangle_{5,6} \otimes \ldots$ we arrive at a state for which $\vec{v}_{1} \cdot \vec{v}_{2}=-\left\|\vec{v}_{1}\right\|\left\|\vec{v}_{2}\right\|$. Thus, this state saturates Eq. (14).

In general, however, the bounds derived by the method above are not sharp. Especially, when we consider frustrated lattices, the bounds are not sharp, and more sophisticated estimates are required. We will discuss one example of a frustrated lattice later in detail. Also, if $N$ is not a multiple of $k$, the bound for $k$-producibility may not be sharp. This is, however, not a major problem. The energy difference between this case and the nearest $N$ which is multiple of $k$ is bounded by a constant. Since we are interested in the thermodynamic limit $N \rightarrow \infty$, and the energy difference per bond decreases as $1 / N$, we can neglect this case.

What is required to derive similar bounds as Eq. (18) for other spin systems and higher degrees of multipartite entanglement? The main ingredient are bounds as in Eqs. (13, 15 16). These bounds depend on the Hamiltonian and on the underlying lattice. For many Hamiltonians and two-qubit blocks, these bounds can straightforwardly be computed analytically. But even if this is not possible, one can simply compute them by numerical minimization over a small number of qubits, if desired, this minimizations can be performed with assurance of global optimality [16].

Finally, the reader should note the difference between the estimation method presented in this Section and the one used in Ref. [10]. The method in Ref. [10] does not separate between factorizing and non-factorizing terms in the Hamiltonian, instead, it estimates the complete Hamiltonian via the Cauchy-Schwarz inequality. As a consequence, it requires more effort and is restricted to one-dimensional systems.

\section{THREE SIMPLE APPLICATIONS}

In this Section, we will apply the presented method to various examples of spin systems. We will first compute energy bounds for $k$-producibility of spin systems with an anti-ferromagnetic Heisenberg interaction in various dimensions. Then we will consider the Ising model and the XX model in an external magnetic field.

\section{A. The Heisenberg model}

Let us first consider the anti-ferromagnetic Heisenberg interaction. That is, we consider the Hamiltonian

$$
H_{H}=\sum_{<i, j>} X_{i} X_{j}+Y_{i} Y_{j}+Z_{i} Z_{j}
$$

on a $D$-dimensional lattice. For this model, we can state:

Theorem 1. (a) Let us consider an infinite onedimensional spin system with the Heisenberg interaction.
Then, the energy bounds per bond for one-, two-, three-, and four-producible states are given by

$$
\begin{gathered}
E_{1 p}^{1 D}=-1 ; \quad E_{2 p}^{1 D}=-\frac{3}{2} \\
E_{3 p}^{1 D}=-1.505 ; \quad E_{4 p}^{1 D}=-1.616 .
\end{gathered}
$$

The ground state energy per bond is known to be $E_{0}=$ $-(4 \ln 2-1) \approx-1.773[17]$, thus all the bounds above are violated by the ground state.

(b) For the two-dimensional square lattice, the respective energies per bond are given by

$$
\begin{gathered}
E_{1 p}^{2 D}=-1 ; \quad E_{2 p}^{2 D}=-\frac{13}{12} \\
E_{3 p}^{2 D}=-1.108 ; \quad E_{4 p}^{2 D}=-1.168 .
\end{gathered}
$$

Here, the energy per bond in the ground state is given by $E_{0}=-1.338$ [7].

(c) For the three-dimensional lattice we have $E_{0}=$ -1.194 [7] and the thresholds for multipartite entanglement read

$$
\begin{gathered}
E_{1 p}^{3 D}=-1 ; \quad E_{2 p}^{3 D}=-\frac{31}{30} \\
E_{3 p}^{3 D}=-1.044 ; \quad E_{4 p}^{3 D}=-1.067 .
\end{gathered}
$$

All the bounds given in this theorem are sharp.

Proof. The proof of this theorem works just as described in the previous section. For the one-producible (i.e. the fully separable) states, the bounds have already been shown before [5, 6, 7]. The bounds for twoproducible states have been obtained analytically (see Lemma 1 in the Appendix); here, the bound for the onedimensional chain was already derived in Ref. [10]. The bounds for the three- and four-qubit case have been obtained numerically [18]. Note that for four-producible states and $D \geq 2$ several possibilities of four-qubit blocks have to be taken into account. The sharpness of the bounds follows also as discussed in the previous section.

\section{B. The Ising model in a transverse magnetic field}

As a second example, let us study the one-dimensional Ising-model in a transverse magnetic field. That is, we consider the Hamiltonian

$$
H_{I}=\sum_{<i, j>} X_{i} X_{j}+B \sum_{i} Z_{i}
$$

The estimation of the energy for $k$-producible states can be performed as in the previous section. Only the interaction terms with the magnetic field have to be added in the definition of $\mathcal{I}(i)$. For instance, for two-qubit blocks, we have to compute

$$
C_{i}=\max _{|\psi\rangle}\left[-x_{k} x_{l}-B\left(z_{k}+z_{l}\right)+\frac{1}{2}\left(x_{k}^{2}+x_{l}^{2}\right)\right] .
$$




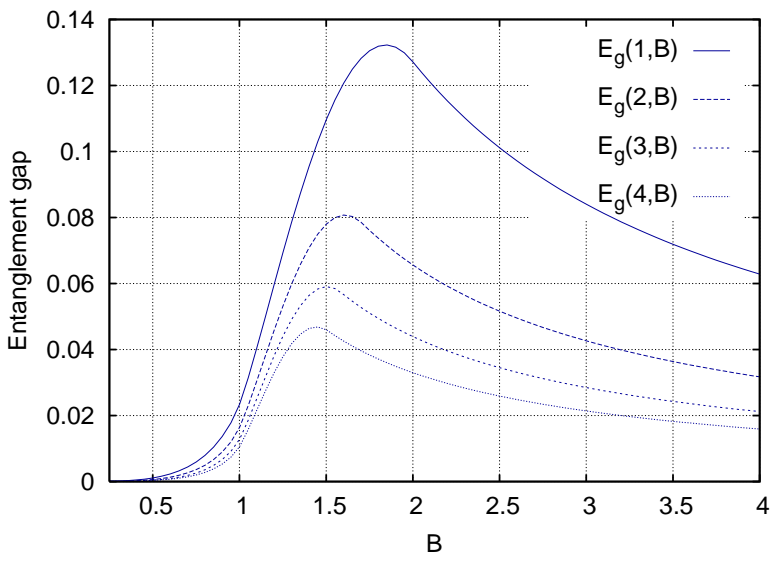

FIG. 3: Entanglement gap $E_{g}(k, B)$ for two- three-, four- and five-partite entanglement for the Ising model in a transverse magnetic field. See text for details.

This and similar maximizations can easily be performed numerically. The resulting bounds are always sharp. Note that the bound for one-producible states has already been derived employing a different method in Refs. [5, 7].

The Ising model is analytically solvable and the thermodynamic properties of the thermal states are known [19]. To investigate the multipartite entanglement properties, we first compute the energy thresholds $E_{k p}$ for kproducible states. We then compare these energies with the ground state energy by calculating the entanglement gap

$$
E_{g}(k, B)=E_{k p}(B)-E_{0}(B),
$$

that is the difference between the ground state energy and the minimal energy for $k$-producible states [7]. Note that the energy minimum for separable states for a quantum Hamiltonian equals the energy minimum of the corresponding classical spin chain $[5]$. Thus $E_{g}(1, B)$ is the energy difference between the classical and the quantum Ising spin chains. The results are shown in Fig. 3.

To discuss these results, let us consider Fig. 3 and look at the curve corresponding to $E_{g}(1, B)$. For a magnetic field slightly larger than $B_{c}=1$ the entanglement gap (and thus the entanglement in the thermal state) is larger than further from this point. Note that at $B_{c}=1$ the ground state of the Ising model undergoes a quantum phase transition. $E_{g}(n, B)$ for $n>1$ also takes its maximum around $B_{c}$. Fig. [3]shows that the field corresponding to this maximum is decreasing with increasing $n$.

Now let us study $F(k, B)=\partial E_{g}(k, B) / \partial B$ as the derivative of $E_{g}(k, B)$ with respect to $B$. These curves are shown in Fig. 4 . On can see directly from this figure that the slope of $F(1, B)$ has an abrupt change at $B_{c, 1}=2$. Further analysis shows, that $F(1, B)$ is also non-analytical at $B_{c}=1$. The non-analytical point $B_{c}$ corresponds to a quantum phase transition of the quantum spin chain, while the change in the slope at $B_{c, 1}=2$

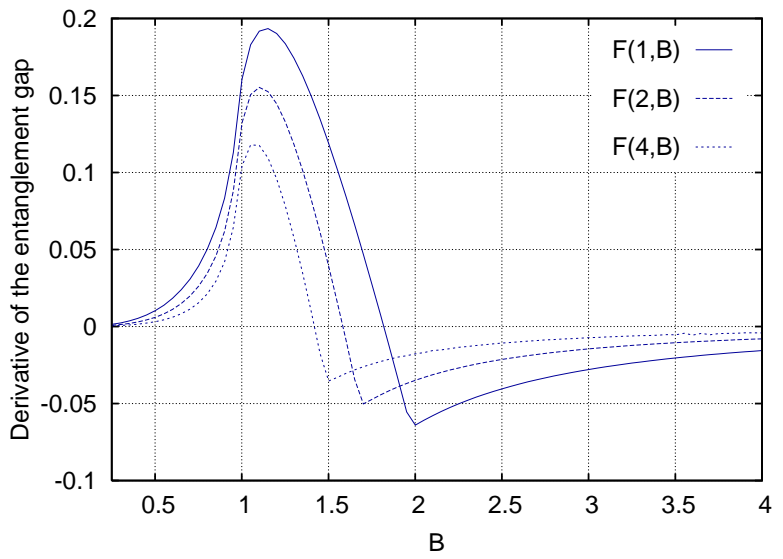

FIG. 4: Derivative of the entanglement gap $F(k, B)=$ $\partial E_{g}(k, B) / \partial B$ for $k=1,2$ and 4 .

corresponds to the critical point of the classical spin system [20].

Similar results can be obtained for $E_{g}(k, B)$ for $k>1$. The energy minimum for $k$-producible states equals also the energy minimum of a spin model in which blocks of $k$ quantum spins interact classically, i.e., in a mean-field fashion [21]. Again there are non-analytical points for $F(k, B)$ at $B_{c}=1$ and at $B_{c, n}>1$. Fig. 4 shows the curves corresponding to $F(1, B), F(2, B)$ and $F(4, B)$. It is clearly visible how $B_{c, k}$ approaches $B_{c}=1$ with increasing $n$. It can also be seen that the maximum of $F(k, B)$ also approaches $B_{c}$ as $k$ increases. A detailed study of the thermodynamics arising from these models intermediate between classical and quantum spin chains will be reported elsewhere.

\section{The XX model in a magnetic field}

As a third example, we study the one-dimensional XX model in a magnetic field. The Hamiltonian of this model is

$$
H_{X X}=\sum_{<i, j>} X_{i} X_{j}+Y_{i} Y_{j}+B \sum_{i} Z_{i}
$$

The estimation of the energy for $k$-producible states can be performed similarly as for the Ising model. Since the XX-model can be solved analytically 22], it is now interesting to investigate the regions in the $T$ - $B$-plane where multipartite entanglement must be present. This has been done in Fig. 5. Similar to the Ising model after the quantum phase transition at $B=2$ the thermal states show different forms of multipartite entanglement, even at relatively high temperatures. 


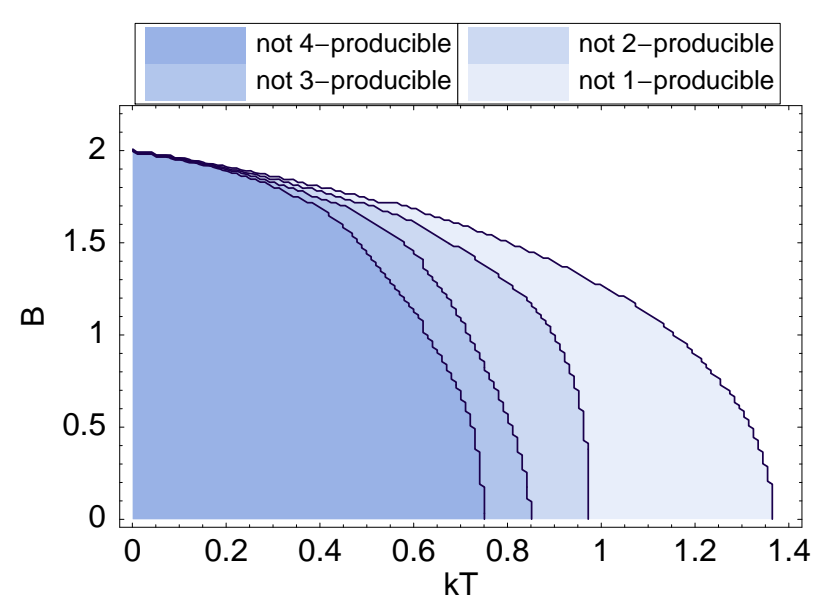

FIG. 5: Entanglement in thermal states of the XX-model in a magnetic field. The regions in the $T$ - $B$-plane are shown where the different types of multipartite entanglement can be detected with our method.

\section{THE HEISENBERG MODEL ON A TRIANGULAR LATTICE}

Let us finally demonstrate with an example that our method with some modifications also allows the computation of energy thresholds for frustrated lattices [23]. Generally, all lattices can be divided into two classes: Bipartite lattices are lattices, where the lattice points can be divided into two sublattices, such that each point in each sublattice interacts only with points which belong to the other sublattice. An example is the twodimensional square lattice, for which these two lattices form a chessboard-like configuration. A lattice is called frustrated if it is not bipartite. This terminology refers to the fact that for such lattices the ground state energy per bond is usually larger than that for two qubits interacting alone.

Entanglement properties of frustrated systems have also been investigated [7, 24]. Concerning our approach, the fact that the ground state energy is large makes it difficult to derive energy bounds for $k$-producible states which are violated by the ground state.

As such an example of a frustrated quantum system we study now the Heisenberg model on a two-dimensional triangular lattice. That is, we consider the Hamiltonian of Eq. (6) on the lattice of Fig. 6. Let us shortly note some properties of this system. The ground state energy per bond in known to be $E_{0}=-0.726$ [14]. From a comparison with a classical spin configuration, it was shown in Ref. 7] that the minimal energy per bond for fully separable (i.e. one-producible) states is $E_{1 p}=-0.5$. Here, we want to derive a bound for two-producible states.

If we apply directly the method of the previous section, the resulting bound is not violated by the ground state. The reason is the following: In the derivation, we used in Eq. (12) the bound $\vec{v}_{1} \cdot \vec{v}_{2} \geq-\left\|\vec{v}_{1}\right\|\left\|\vec{v}_{2}\right\|$. This bound is

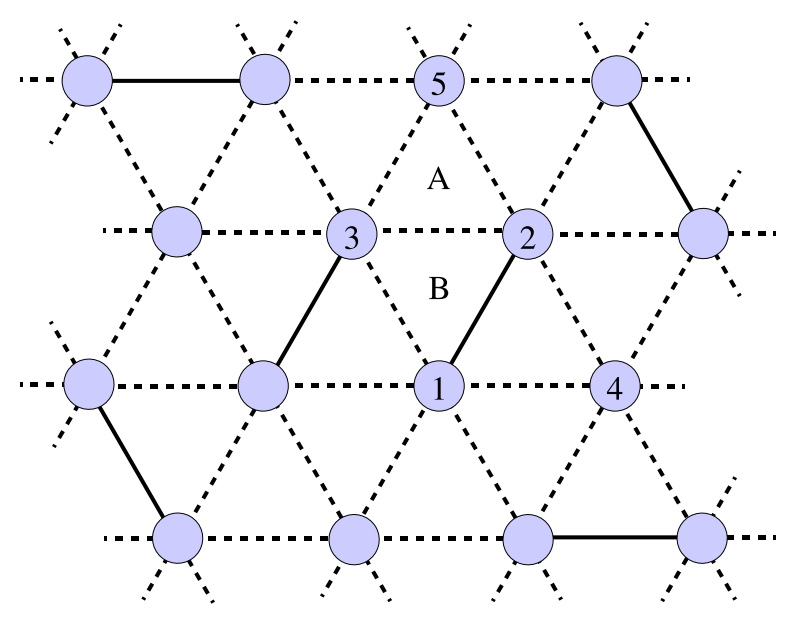

FIG. 6: A two-producible state on a triangular lattice. Solid lines represent possible entanglement between the qubits. The triangle $(2,5,3)$ is type A, i.e., it does not have entanglement between its qubits. The triangle $(1,2,3)$ is of type $\mathrm{B}$ since qubits 1 and 2 may be entangled. When estimating $C_{i}$ for the block of the qubits 1 and 2 , the two triangles $(1,2,3)$ and $(1,2,4)$ are estimated via Eq. (31), since there are definitely of type B. See text for further details.

not sharp for frustrated lattices. Thus, we have to make a more sophisticated estimate.

First, note that the scalar product $\vec{v}_{1} \cdot \vec{v}_{2}$ represents all factorizing terms in the Hamiltonian. These terms can be grouped into the contributions corresponding to different triangles $T_{i}$. So we can write

$$
2 \vec{v}_{1} \cdot \vec{v}_{2}=\sum_{\text {triangles }} \mathfrak{h}\left(T_{i}\right)
$$

where the triangle contributions $\mathfrak{h}\left(T_{i}\right)$ can be of two types, depending on the triangle: For a triangle on the qubits $j, k, l$ with no entanglement between the qubits $j, k, l$ (type A triangle, see Fig. 6) we have

$$
\mathfrak{h}\left(T_{i}\right)=\sum_{a=x, y, z}\left(a_{j} \cdot a_{k}+a_{k} \cdot a_{l}+a_{l} \cdot a_{j}\right) .
$$

For triangles where two of the three qubits (say, $k$ and $l$ ) may be entangled (type B) we have

$$
\mathfrak{h}\left(T_{i}\right)=\sum_{a=x, y, z}\left(a_{j} \cdot a_{k}+a_{j} \cdot a_{l}\right)
$$

The prefactor of two in Eq. (27) stems from the fact that every bond contributes to two triangles.

Now we need the facts that

$-\left(\frac{a_{j}}{\sqrt{2}} \cdot \frac{a_{j}}{\sqrt{2}}+\frac{a_{k}}{\sqrt{2}} \cdot \frac{a_{k}}{\sqrt{2}}+\frac{a_{l}}{\sqrt{2}} \cdot \frac{a_{l}}{\sqrt{2}}\right) \leq a_{j} \cdot a_{k}+a_{k} \cdot a_{l}+a_{l} \cdot a_{j}$,

which holds for all real numbers $a_{j}, a_{k}, a_{l}$, and we need the estimate

$$
-\left(\frac{a_{k} a_{l}+1}{\sqrt{2}} \cdot \frac{a_{j}}{\sqrt{2}}+\frac{a_{k} a_{l}+1}{\sqrt{2}} \cdot \frac{a_{j}}{\sqrt{2}}\right) \leq a_{k} \cdot a_{j}+a_{l} \cdot a_{j} .
$$


This estimate holds since $a_{i}$ and $a_{k} a_{l}$ are expectation values of (tensor products) of Pauli matrices. Namely, from the positivity of the density matrix the relation $-\left(1+a_{k} a_{l}\right) \leq a_{k}+a_{l} \leq\left(1+a_{k} a_{l}\right)$ follows, which results in Eq. (31).

The bounds in Eq. (30, 31) can be interpreted in the following way: for each of the $\mathfrak{h}\left(T_{i}\right)$ there are two vectors $\vec{w}_{i}^{(1)}$ and $\vec{w}_{i}^{(2)}$ such that

$$
\mathfrak{h}\left(T_{i}\right) \geq-\vec{w}_{i}^{(1)} \cdot \vec{w}_{i}^{(2)} .
$$

If $T_{i}$ is of the type A then $\vec{w}_{i}^{(1)}$ has nine entries and if $T_{i}$ is of the type B it has six entries. Note that the definition of $\vec{w}_{i}^{(1)}$ etc. does not directly correspond to the $\mathcal{R}$ and $\mathcal{N}$ in the definition of $\vec{v}_{1 / 2}$.

Thus, if we define

$$
\vec{W}^{(1)}=\bigoplus_{i} \vec{w}_{i}^{(1)}, \quad \vec{W}^{(2)}=\bigoplus_{i} \vec{w}_{i}^{(1)},
$$

it follows that $2 \vec{v}_{1} \cdot \vec{v}_{2} \geq-\vec{W}^{(1)} \cdot \vec{W}^{(2)}$. Using the CauchySchwarz inequality yields

$$
\vec{v}_{1} \cdot \vec{v}_{2} \geq-\frac{1}{4}\left(\left\|\vec{W}^{(1)}\right\|^{2}+\left\|\vec{W}^{(2)}\right\|^{2}\right) .
$$

The right hand side of Eq. (34) is a sum of many squares of expectation values of one- or two qubit observables. Each of these expectation values originates from a certain block. By counting carefully the contributions of each block, we can now estimate them separately for each block.

A one-qubit block on the qubit $k$, contributes to the estimates of six triangles. These may be triangles of type A or B. An estimate of one triangle of the type A results in two times a contribution $\left(a_{k} / \sqrt{2}\right)^{2}$ in $\left\|\vec{W}^{(1)}\right\|^{2}+\left\|\vec{W}^{(2)}\right\|^{2}$, one in $\left\|\vec{W}^{(1)}\right\|^{2}$ and one in $\left\|\vec{W}^{(2)}\right\|^{2}$ (see Eq. (30)). A triangle of the type $\mathrm{B}$ gives also two times this contribution, but now either two times in $\left\|\vec{W}^{(1)}\right\|^{2}$ or two times in $\left\|\vec{W}^{(2)}\right\|^{2}$ (see Eq. (31)). Thus, in analogy to Eqs. 113 15), we have to estimate

$$
C_{i}=\max _{|\psi\rangle}\left[\frac{1}{4} \cdot 6 \cdot 2\left(\frac{x_{k}^{2}}{2}+\frac{y_{k}^{2}}{2}+\frac{z_{k}^{2}}{2}\right)\right]=\frac{3}{2} .
$$

A two-qubit block on the qubits $k$ and $l$ contributes to ten triangles. Two of them contain both the qubits $k$ and $l$ and are thus of the type B (see Fig. 6). Each of them contribute two times $\left(a_{k} a_{l}+1 / \sqrt{2}\right)^{2}$ to $\left\|\vec{W}^{(1)}\right\|^{2}+$ $\left\|\vec{W}^{(2)}\right\|^{2}$, either both in $\left\|\vec{W}^{(1)}\right\|^{2}$ or both in $\left\|\vec{W}^{(2)}\right\|^{2}$ (see Eq. (31)). For the other eight triangles, it does not matter as for the one-qubit blocks whether they are of the type A or B. Thus, we have to estimate

$$
\begin{aligned}
C_{i} & =\max _{|\psi\rangle}\left[-x_{k} x_{l}-y_{k} y_{l}-z_{k} z_{l}+\right. \\
& +\frac{2 \cdot 2}{4}\left(\frac{\left(1+x_{k} x_{l}\right)^{2}}{2}+\frac{\left(1+y_{k} y_{l}\right)^{2}}{2}+\frac{\left(1+z_{k} z_{l}\right)^{2}}{2}\right) \\
& \left.+\frac{4 \cdot 2}{4}\left(\frac{x_{k}^{2}}{2}+\frac{y_{k}^{2}}{2}+\frac{z_{k}^{2}}{2}+\frac{x_{l}^{2}}{2}+\frac{y_{l}^{2}}{2}+\frac{z_{l}^{2}}{2}\right)\right]=4 .
\end{aligned}
$$

This follows similarly as before, see also Lemma 2(b) in the Appendix.

With these bounds we have for the two-producible state $\langle H\rangle \geq-\sum_{i} C_{i}$, which finally results in a minimal energy per bond of

$$
E_{2 p}=-\frac{2}{3},
$$

which is clearly violated by the ground state. Thus, at a considerable temperature, in a frustrated triangular Heisenberg lattice the thermal state is not two-producible and the effects of multipartite entanglement cannot be neglected.

Finally, it should be noted that it is not clear whether the bound in Eq. (37) is sharp. Two facts suggest that this is not the case. First, the use of the Cauchy-Schwarz inequality in Eq. (34) is probably not sharp, since all the vectors $\vec{W}^{(1)}$ and $\vec{W}^{(2)}$ are usually not parallel. Second, the maximum in Eq. (36) is obtained for a separable state, no entanglement is required to saturate this bound.

\section{CONCLUSION}

In conclusion, we have developed a method to investigate the presence of multipartite entanglement in spin models. Our method relies on energy bounds for certain classes of multipartite entangled states and can be applied to any state, not only to thermal states. We discussed different examples and showed that our ideas can in principle also be applied to the investigation of frustrated systems.

The results of this paper may be applied in several directions. On the one hand, based on our energy thresholds one may derive temperature bounds below which entanglement must be present. Then, one may try to design methods to extract this entanglement and make it useful for some tasks.

On the other hand, our results can also be used to gain theoretical insight concerning the validity of ground state approximations. For example, one might be interested in the ground state energy $E_{0}$. To approximate this, one may consider some mean-field like approximation, where the trial wave function is a product state with respect to all qubits 25. This corresponds to a one-producible state. Concerning the justification of this approximation, we can say with our method the following: If $E_{1 p} \approx E_{2 p}$ the approximation might be justified, while if $E_{1 p} \gg E_{2 p}$ this is clearly not the case.

\section{ACKNOWLEDGMENT}

We would like to thank H.J. Briegel, J.I. Cirac, M. Dowling, W. Dür, O. Gittsovich, A. Hamma, P. Hyllus, D. Porras, J. Richert, E. Rico and T. Roscilde for useful discussions. We also acknowledge the support of the EU (IST-2001-38877, OLAQUI, ProSecCo, QUPRODIS, 
RESQ, SCALA) the FWF, the DFG and the Kompetenznetzwerk Quanteninformationsverarbeitung der Bayerischen Staatsregierung. G.T. thanks the support of the European Union (Grant Nos. MEIF-CT-2003500183 and MERG-CT-2005-029146) and National Research Fund of Hungary OTKA under Contract No. T049234.

\section{APPENDIX}

Here, we prove two useful bounds for our proofs.

Lemma 1. For two-qubit state $|\psi\rangle$ on the qubits $k, l$ and $\gamma \geq 1 / 2$ we have the following sharp bound:

$$
\begin{aligned}
& -x_{k} x_{l}-y_{k} y_{l}-z_{k} z_{l}+\gamma\left(x_{k}^{2}+y_{k}^{2}+z_{k}^{2}+x_{l}^{2}+y_{l}^{2}+z_{l}^{2}\right) \\
& \quad \leq 1+2 \gamma+\frac{1}{2 \gamma} .
\end{aligned}
$$

Proof. We have to maximize the left hand side of the inequality over all states. First note that a generic quantum state can be written as

$$
|\psi\rangle\langle\psi|=\frac{1}{4} \sum_{i, j=1, x, y, z} \lambda_{i j} \sigma_{i} \otimes \sigma_{j},
$$

with $\sigma_{1}=\mathbb{1}$. Here, $\lambda_{i j}=\left\langle\psi\left|\sigma_{i} \otimes \sigma_{j}\right| \psi\right\rangle$ holds, thus we can directly maximize over all allowed $\lambda_{i j}$. Let us first consider local unitary transformations. These transformations act on $\lambda_{i j}$ as $\left(\lambda_{i j}\right) \rightarrow\left(1 \oplus O_{L}\right)\left(\lambda_{i j}\right)\left(1 \oplus O_{R}\right)$ where $O_{L}$ and $O_{R}$ are orthogonal $3 \times 3$ matrices. Here, $1 \oplus O_{R}$ denotes a $4 \times 4$ matrix with a block structure, i.e., with " 1 " in the left upper corner and $O_{R}$ denotes the $3 \times 3$ block in the right bottom corner. These transformations do not change the purities of the reduced states, thus they do not change $x_{k}^{2}+y_{k}^{2}+z_{k}^{2}$ and $x_{l}^{2}+y_{l}^{2}+z_{l}^{2}$. Furthermore, $\left|x_{k} x_{l}\right|+\left|y_{k} y_{l}\right|+\left|z_{k} z_{l}\right|$ is the sum of the absolute values of the diagonal elements of the $3 \times 3$ submatrix $\lambda_{i j}^{r e d}=\lambda_{i, j=x, y, z}$. This sum is maximized when $\lambda^{\text {red }}$ is brought to diagonal form via a singular value decomposition. This decomposition can be performed by local unitary operations.

Thus it suffices to consider $|\psi\rangle=\alpha|00\rangle+\beta|11\rangle$ with $\alpha^{2}+\beta^{2}=1$, since for that state we have

$$
\left(\lambda_{i j}\right)=\left(\begin{array}{cccc}
1 & 0 & 0 & \alpha^{2}-\beta^{2} \\
0 & 2 \alpha \beta & 0 & 0 \\
0 & 0 & -2 \alpha \beta & 0 \\
\alpha^{2}-\beta^{2} & 0 & 0 & 1
\end{array}\right) .
$$

The final maximization over all $\alpha$ can then be directly performed. The left hand side of Eq. (38) is maximized for $4 \alpha^{2}=2-\sqrt{4 \gamma^{2}-1} / \gamma$ which proves the bound in Eq. (38).

Lemma 2. (a) For two-qubit state $|\psi\rangle$ on the qubits $k, l$ and $\gamma \geq 1 / 2$ we have the following sharp bound:

$$
-x_{k} x_{l}-y_{k} y_{l}+\gamma\left(x_{k}^{2}+y_{k}^{2}+x_{l}^{2}+y_{l}^{2}\right) \leq 1+2 \gamma+\frac{1}{8 \gamma} \text {. }
$$

(b) Similarly, we have

$$
\begin{aligned}
& \frac{3}{2}+\frac{1}{2}\left(x_{k} x_{l}^{2}+y_{k} y_{l}^{2}+z_{k} z_{l}^{2}\right)+ \\
& +\left(x_{k}^{2}+y_{k}^{2}+z_{k}^{2}+x_{l}^{2}+y_{l}^{2}+z_{l}^{2}\right) \leq 4 .
\end{aligned}
$$

Proof. (a) For the minimization it does not matter whether we minimize over the observables $X, Y$ [as in Eq. (41)] or $X, Z$. Then, the bound can be derived as in the proof of Lemma 1. One arrives again at Eq. (40), now only one of the terms $2 \alpha \beta$ has to be omitted in the final maximization. (b) This can also be derived as in Lemma 1. When applying local unitary transformations, $\left(\left(x_{k} x_{l}\right)^{2}+\left(y_{k} y_{l}\right)^{2}+\left(z_{k} z_{l}\right)^{2}\right)$ is maximal, when the $3 \times 3$ matrix is diagonal. Finally, one has only to maximize over $\alpha$ again.
[1] For general results on entanglement in condensed matter systems see A. Osterloh, L. Amico, G. Falci, and R. Fazio, Nature 416, 608 (2002); T.J. Osborne and M.A. Nielsen, Phys. Rev. A 66, 032110 (2002); G. Vidal, J.I. Latorre, E. Rico, and A. Kitaev, Phys. Rev. Lett. 90, 0227902 (2003); V.E. Korepin, Phys. Rev. Lett. 92, 096402 (2004); F. Verstraete, M. Popp, and J.I. Cirac, Phys. Rev. Lett. 92, 027901 (2004); H. Fan, V.E. Korepin and V. Roychowdhury, Phys. Rev. Lett. 93, 227203 (2004); M.B. Plenio, J. Eisert, J. Dreißig, and M. Cramer, Phys. Rev. Lett. 94060503 (2005).

[2] For results concerning multipartite entanglement in spin models see X. Wang, Phys. Rev. A 66, 044305 (2002); L.F. Santos, Phys. Rev. A 67, 062306 (2003); P. Štelmachovič and V. Bužek, Phys. Rev. A 70, 032313 (2004); T. Roscilde, P. Verrucchi, A. Fubini, S. Haas, and V. Tognetti, Phys. Rev. Lett. 93, 167203 (2004);
Phys. Rev. Lett. 94, 147208 (2005); T.-C. Wei, D. Das, S. Mukhopadyay, S. Vishveshwara, and P.M. Goldbart, Phys. Rev. A 71, 060305(R) (2005); D. Bruß, N. Datta, A. Ekert, L.C. Kwek, and C. Macchiavello, Phys. Rev. A 72, 014301 (2005).

[3] For a studies on entanglement dynamics in spin systems see for example L. Amico, A. Osterloh, F. Plastina, R. Fazio, and G.M. Palma, Phys. Rev. A. 69, 022304 (2004); M. Koniorczyk, P. Rapcan, and V. Bužek, Phys. Rev. A 72, 022321 (2005); A. Sen(De), U. Sen, and M. Lewenstein, quant-ph/0505006

[4] G. Vidal, Phys. Rev. Lett. 93, 040502 (2004); F. Verstraete, D. Porras and J.I. Cirac, Phys. Rev. Lett. 93, 227205 (2004); U. Schollwöck, Rev. Mod. Phys. 77, 259 (2005).

[5] G. Tóth, Phys. Rev. A 71, 010301(R) (2005).

[6] Č. Brukner and V. Vedral, quant-ph/0406040 
[7] M.R. Dowling, A.C. Doherty, S.D. Bartlett, Phys. Rev. A 70, 062113 (2004).

[8] For other works relating the energy to entanglement see A.N. Jordan and M. Büttiker, Phys. Rev. Lett. 92, 247901 (2004); L.-A. Wu, S. Bandyopadhyay, M.S. Sarandy, and D.A. Lidar, Phys. Rev. A 72, 032309 (2005).

[9] For works relating entanglement to other macroscopic observables, see, e.g. S. Ghosh, T.F. Rosenbaum, G. Aeppli and S.N. Coppersmith, Nature (London) 425, 48 (2003); I. Bose and A. Tribedi, Phys. Rev. A 72, 022314 (2005); Č. Brukner, V. Vedral, and A. Zeilinger, quant-ph/0410138 M. Wiesniak, V. Vedral and Č. Brukner, New J. Phys. 7, 258 (2005); T. Vértesi and E. Bene, cond-mat/0503726

[10] O. Gühne, G. Tóth and H.J. Briegel, New J. Phys. 7, 229 (2005).

[11] Note that this is also true for the classification concerning $k$-separability or the classification of $W / G H Z$ states for three qubits.

[12] A. Acín, D. Bruß, M. Lewenstein and A. Sanpera, Phys. Rev. Lett. 87, 040401 (2001).

[13] J. Eisert and H.J. Briegel, Phys. Rev. A 64, 022306 (2001).

[14] C. Lhuillier and G. Misguich, cond-mat/0109146
[15] In our example, one may also prove this explicitly and take the two-qubit state $|\phi\rangle\langle\phi|=\left(\mathbb{1} \otimes \mathbb{1}+2 a \sqrt{1-a^{2}}\left(\sigma_{x} \otimes\right.\right.$ $\left.\left.\sigma_{x}+\sigma_{y} \otimes \sigma_{y}\right)-\sigma_{z} \otimes \sigma_{z}-\left(2 a^{2}-1\right)\left(\sigma_{z} \otimes \mathbb{1}-\mathbb{1} \otimes \sigma_{z}\right)\right) / 4$ with $a=-\sqrt{1 / 2-\sqrt{2} / 3}$, which saturates the bound in Eq. (16) to find the total state saturating Eq. (18).

[16] J. Eisert, P. Hyllus, O. Gühne, and M. Curty, Phys. Rev. A 70, 062317 (2004).

[17] L. Hulthén, Arkiv Math. Astron. Fys. 26A 1 (1938).

[18] For all our numerical computations we used the optimization packages of the computer algebra system "Mathematica".

[19] P. Pfeuty, Ann. Phys. (N.Y.) 57, 79 (1970).

[20] Diego Porras, private communication.

[21] An example for this type of mixed quantum-classical system can be found in G. Tóth and C. S. Lent, J. Appl. Phys. 89, 7943 (2001).

[22] S. Katsura, Phys. Rev. 127, 1508 (1962); erratum: ibid., 129, 2835 (1963).

[23] For an overview, see H.T. Diep (ed.), "Frustrated spin systems", (World-Scientific, Singapore, 2005); R. Moessner, Can. J. Phys. 79, 1283 (2001).

[24] C.M. Dawson and M.A. Nielsen, Phys. Rev. A 69, 052316 (2004).

[25] See, e.g. J. Richert, cond-mat/0510343 